MATHEMATICS OF COMPUTATION

Volume 69, Number 230, Pages 749-756

S $0025-5718(99) 01113-8$

Article electronically published on May 21, 1999

\title{
A NEW SUMMATION METHOD FOR POWER SERIES WITH RATIONAL COEFFICIENTS
}

\author{
SILVIA DASSIÈ, MARCO VIANELLO, AND RENATO ZANOVELLO
}

\begin{abstract}
We show that an asymptotic summation method, recently proposed by the authors, can be conveniently applied to slowly convergent power series whose coefficients are rational functions of the summation index. Several numerical examples are presented.
\end{abstract}

\section{Asymptotic summation}

Consider a power series

$$
\sum_{j=1}^{\infty} f_{j} z^{j}
$$

where $z \in \mathbb{C}$ and $f_{j} \in \mathbb{C}$. In 3 we proved that, when the coefficients $f_{j}$ possess an asymptotic expansion

$$
f_{j} \sim a_{1} j^{-p_{1}}+a_{2} j^{-p_{2}}+\ldots, j \rightarrow \infty, 0<p_{1}<p_{2}<\ldots,
$$

and $p_{k+1}-p_{k} \in \mathbb{N}$, then also the remainder of (1) has an asymptotic expansion

$$
\sum_{j=n}^{\infty} f_{j} z^{j} \sim z^{n} n^{-q}\left\{b_{1}(z) n^{-1}+b_{2}(z) n^{-2}+\cdots\right\}, n \rightarrow \infty,
$$

where $q=p_{1}-1$ if $|z| \leq 1$ and $z \neq 1$, and $q=p_{1}-2$ if $z=1$. In particular, we provided explicit formulas for the computation of the coefficients $b_{k}(z)$, which allow for a direct use of expansion (3) for the numerical summation of (2). It is worth recalling that in [11] Sidi already showed the mere existence of an asymptotic expansion for the remainder, as a basis for acceleration of convergence by means of Levin's T-transformation. Convergence of the series (2), in fact, can be very slow (when $p_{1}$ is "small") for $z$ on (or close to) the boundary of the unit circle.

When $p_{k+1}-p_{k} \equiv 1$, which is the most common case in applications, the quoted formulas are simple and easy to implement. In fact, for $z \neq 1$ we have

$$
b_{k}(z)=-\sum_{i=0}^{k-1} a_{k-i} \varphi_{i}(k, z)
$$

Received by the editor April 28, 1998.

1991 Mathematics Subject Classification. Primary 65B10, 65D20; Secondary 33E20, 41A60.

Key words and phrases. Slowly convergent power series, rational coefficients, asymptotic expansions, asymptotic summation.

Work supported, in part, by the Italian MURST, and by the GNIM-CNR.

(C)2000 American Mathematical Society 
where the $\varphi_{i}$ are recursively defined by

$$
\begin{gathered}
\varphi_{0}(k, z)=\frac{1}{z-1}, \\
\varphi_{i}(k, z)=\frac{z}{1-z} \sum_{r=0}^{i-1}\left(\begin{array}{c}
i+1-k-p_{1} \\
i-r
\end{array}\right) \varphi_{r}(k, z), \quad i=1,2, \ldots,
\end{gathered}
$$

while for $z=1$ we have

$$
b_{k}(1)=\sum_{i=1}^{k} \frac{a_{i}}{p_{1}+i-2}\left(\begin{array}{c}
2-p_{1}-i \\
k-i
\end{array}\right) B_{k-i},
$$

where $B_{k}$ are the Bernoulli numbers; cf. [2], [3, Formula (24)]. Observe that the more general formula (5) of [3] contains a misprint: the number $k+p_{\ell}-p_{1}-1$ in the binomial coefficient, should be $k+p_{1}-p_{\ell}-1$ instead.

As a numerical application, we considered in 3 the evaluation of the special function

$$
\begin{gathered}
S\left(z ; j_{0}, \nu, a, b, p\right)=\sum_{j=j_{0}}^{\infty} z^{j}(j+b)^{\nu-1}(j+a)^{-p},|z| \leq 1, \\
0<\nu \leq 1, \quad a, b \in \mathbb{C} \backslash\left\{-j_{0},-j_{0}-1, \ldots\right\},
\end{gathered}
$$

generalizing various summation problems that appeared in the recent literature on convergence acceleration of numerical and power series; cf., e.g., [1, 4, 5, 6, 8, 9, 10, When $z$ is on or close to the boundary of the unit circle, summation of (7) was accomplished by a numerical algorithm based directly on the expansion (3) and the formulas (4)-(6).

\section{The CASE of RATiOnal COEFFICIEnts}

In the present work, we show that the approach briefly described in the previous section can be also applied to the numerical summation of series whose coefficients are rational functions of the index, i.e.,

$$
\begin{gathered}
S=S(z)=\sum_{j=j_{0}}^{\infty} z^{j}(j+b)^{\nu-1} r(j),|z| \leq 1, \\
0<\nu \leq 1, \quad b \in \mathbb{C} \backslash\left\{-j_{0},-j_{0}-1, \ldots\right\}, r(j)=\frac{\alpha(j)}{\beta(j)}=\frac{\alpha_{s} j^{s}+\cdots+\alpha_{0}}{\beta_{t} j^{t}+\cdots+\beta_{0}},
\end{gathered}
$$

where $t-s>\nu$ if $z=1, t-s>\nu-1$ if $|z| \leq 1$ and $z \neq 1$, and $\beta(j) \neq 0$ for all integers $j \geq j_{0}$.

A possible approach to the summation of (8) in instances of slow convergence is given by partial fraction decomposition of the rational function, together with suitable algorithms for the computation of (7). The series involved in the partial fractions are indeed of that form, with $p \in \mathbb{N}$. This represents a motivation for the development of special summation methods for the series in (7), as pointed out in several recent papers (cf. [4, 6, 8]). On the other hand, though the basic step of partial fraction decomposition can be accomplished in an efficient manner (cf. [7] $\S 7.1])$, it is still quite costly.

For this reason, it is worth pursuing a direct use of asymptotic summation via (3)(5), provided an asymptotic expansion like (2) exists for the coefficient $(j+b)^{\nu-1} r(j)$ and can be easily constructed. This is indeed the case for a rational function 
$r(z)=\alpha(z) / \beta(z)$, which admits an asymptotic expansion in powers of $z^{-1}$, the convergent Laurent expansion, in a suitable neighborhood of $\infty$ which omits the poles of $r(z)$. Thus the following asymptotic relation holds:

$$
\alpha(j)=j^{s-t} \beta(j)\left(\sum_{k=0}^{m} c_{k} j^{-k}+O\left(j^{-m-1}\right)\right), m=0,1,2, \ldots, \quad j \geq \tilde{n},
$$

where $\tilde{n}$ is the least positive integer such that $r(z)$ has no poles for $|z| \geq \tilde{n}$, and the $c_{k}$ 's are the coefficients of the Laurent expansion of $r(z)$.

From (9) we can compute recursively the coefficients $c_{k}$ by means of the $\alpha_{i}$ 's and $\beta_{i}$ 's (cf. (8) ). In fact, substituting from (8) the explicit expressions for $\alpha(j)$ and $\beta(j)$ and comparing the coefficients of like powers of $j$ on the left and right of (9), we obtain the infinite triangular system

$$
\sum_{k=0}^{\ell} c_{k} \beta_{t-\ell+k}= \begin{cases}\alpha_{s-\ell} & \text { if } 0 \leq \ell \leq s \\ 0 & \text { if } \ell>s\end{cases}
$$

and, by forward substitution, we find that

$$
c_{k}= \begin{cases}\left(\alpha_{s-k}-\sum_{i=0}^{k-1} c_{i} \beta_{t-k+i}\right) / \beta_{t} & \text { if } 0 \leq k \leq s, \\ -\sum_{i=0}^{k-1} c_{i} \beta_{t-k+i} / \beta_{t} & \text { if } k>s .\end{cases}
$$

In this way we get the asymptotic expansion

$$
f_{j}:=(j+b)^{\nu-1} r(j)=\sum_{k=1}^{m} a_{k} j^{-p_{k}}+O\left(j^{-p_{m+1}}\right), m=1,2, \ldots, j \geq n^{*},
$$

with

$$
a_{k}=\sum_{i=1}^{k}\left(\begin{array}{c}
\nu-1 \\
k-i
\end{array}\right) c_{i-1} b^{k-i}, p_{k}=k+t-s-\nu, \quad k=1,2, \ldots
$$

and

$$
n^{*}= \begin{cases}\tilde{n} & \text { if } b \in \mathbb{C} \backslash\left(-\infty,-j_{0}\right], \\ \max \{\tilde{n},[|b|]+1\} & \text { if } b \in\left(-\infty, j_{0}\right] \backslash\left\{-j_{0},-j_{0}-1, \ldots\right\} .\end{cases}
$$

In (13), indeed, $a_{k}$ is the $k$ th coefficient of the product of the asymptotic expansions for $r(j)$ and $(j+b)^{\nu-1}$. The fact that $(j+b)^{\nu-1}=j^{\nu-1}(1+b / j)^{\nu-1}$ possesses an asymptotic expansion in powers of $j^{-1}$ is a trivial consequence of the Maclaurin formula for the binomial function $(1+w)^{\nu-1}$ when $w=b / j \in \mathbb{C} \backslash(-\infty,-1]$ (where $j>|b|$ when $b<0)$.

From (3)-(6) we can finally write the representation

$$
\begin{aligned}
& S=S_{n}+R_{n}=\sum_{j=j_{0}}^{n-1} z^{j}(j+b)^{\nu-1} r(j)+\sigma_{n, m}+\varepsilon_{n, m}, m=1,2, \ldots, \\
& \sigma_{n, m}=z^{n} n^{-q} \sum_{k=1}^{m} b_{k}(z) n^{-k}, \quad \varepsilon_{n, m}=z^{n} O\left(n^{-(m+q+1)}\right), n \geq n^{*},
\end{aligned}
$$

where $q=t-s-\nu$ if $z \neq 1$, and $q=t-s-\nu-1$ if $z=1$. 


\section{The SUMmation ALgORITHM}

The representation (15) gives rise to the problem of constructing an algorithm that finds a pair $(n, m)$ such that the relative error $\left|\varepsilon_{n, m} / S\right|$ is below a given tolerance and at the same time the computational cost is low. Recall that, because of the asymptotic nature of the approximation in (15), the error term $\varepsilon_{n, m}$ tends to 0 as $n \rightarrow \infty$, but is not infinitesimal as $m \rightarrow \infty$; indeed, $\left|\varepsilon_{n, m}\right|$ exhibits for each fixed value of $n$ a minimum in $m$ which is infinitesimal as $n \rightarrow \infty$. As in [3], however, we forgo a procedure based on finding (empirically) such a minimum for a given subsequence of indices $\left\{n_{s}\right\}$ : in fact, the complexity in the computation of $\sigma_{n, m}$ increases quadratically $(z=1)$ or cubically $(z \neq 1)$ with $m$, so that this approach turns out to be quite costly.

An alternative strategy, which is that adopted in [3], consists in trying to balance the computational cost of the partial sum $S_{n}$ with that of $\sigma_{n, m}$ in terms of the order of magnitude of the respective flops number. While the computation of $\sigma_{n, m}$ requires basically only elementary arithmetic operations, that of $S_{n}$ may involve the evaluation of logarithms (when $\nu<1$ ). In this regard we have assumed that the computation of a logarithm corresponds to 10 flops: this value was obtained by averaging the results obtained with various programming languages and compilers, on various processors.

The summation algorithm can then be summarized as follows:

(i) $k:=1, n_{0}:=j_{0}-1, S_{n_{0}}:=0, c_{0}:=\alpha_{s} / \beta_{t}$;

(ii) compute the coefficient $a_{k}$ by (11), (13), and $b_{k}(z)$ by (4)-(6);

(iii) compute the partial sum $S_{n_{k}}$ as

$$
S_{n_{k}}:=S_{n_{k-1}}+\sum_{j=n_{k-1}+1}^{n_{k}} z^{j}(j+b)^{\nu-1} r(j)
$$

where $n_{k}$ is choosen in such a way as to balance the cost of $(i i)$ with the cost of (16), as described above;

(iv) for $i=1, \ldots, k$

- compute $\sigma_{n_{k}, i}$ as in (15);

- if $i<k$, then if

$$
\begin{gathered}
\mu_{k, i}\left|\frac{\varepsilon_{n_{k}, i}}{S}\right| \cong\left|\frac{S_{n_{k}}-S_{n_{k-1}}+\sigma_{n_{k}, i}-\sigma_{n_{k-1}, i}}{S_{n_{k}}+\sigma_{n_{k}, i}}\right| \leq \mu_{k, i} \cdot \text { reltol } \\
\text { where } \mu_{k, i}=\left|1-z^{n_{k-1}-n_{k}}\left(\frac{n_{k}}{n_{k-1}}\right)^{q+i+1}\right|
\end{gathered}
$$

then EXIT;

(v) go to step (ii) with $k:=k+1$.

A key observation is now in order. In principle, representation (15) can be used only for $n \geq n^{*}$ (cf. (14)), so that in step (i) one should choose $n_{0} \geq n^{*}$ and compute the initial sum $S_{n_{0}}$ to start the iterative process, making an appropriate use of the a posteriori estimate (17). Such an estimate is strongly based on the asymptotic nature of (15): in fact by (15)) we obtain $\varepsilon_{n, i} \cong z^{n} b_{i+1}(z) n^{-(q+i+1)}$ for 
$n>n^{*}$ sufficiently large, and moreover, we have

$$
\begin{aligned}
& \left|S_{n_{k}}-S_{n_{k-1}}+\sigma_{n_{k}, i}-\sigma_{n_{k-1}, i}\right|=\left|\varepsilon_{n_{k}, i}-\varepsilon_{n_{k-1}, i}\right| \\
& \cong\left|\left(1-z^{n_{k-1}-n_{k}}\left(\frac{n_{k}}{n_{k-1}}\right)^{q+i+1}\right) \varepsilon_{n_{k}, i}\right|
\end{aligned}
$$

for $k$ sufficiently large.

This implies that a (possibly sharp) overestimate of $\tilde{n}$ should be produced (cf. (9)), for example by using for the denominator $\beta(z)$ one of the well-known a priori bounds of the complex zeros of a polynomial, or by applying a suitable localization algorithm for the zeros; cf. [7, Ch.6] for an extensive treatment of these topics. In fact, in excluding the zeros of $\beta(z)$ we exclude a fortiori the poles of $r(z)$.

In practice, however, the summation algorithm sketched above turns out to be "robust" in the sense that it gives a correct answer also starting from $n_{0}=j_{0}-1$, $S_{n_{0}}=0$ : our numerical experiments confirmed that in every case the exit index $n_{k}$ is greater than $n^{*}$. On the other hand, it should also be noticed that this dependence on the value of $n^{*}$ can represent a possible drawback of the method, when $b \ll-1$ or when $r(z)$ exhibits poles with very large modulus.

\section{Numerical EXAMPLES}

In this section we present the results of the application of the asymptotic summation method to several series of the form (8). In all the examples we set a relative tolerance reltol $=10^{-14}$ in the termination test (17): in Tables I-X below $(n, i)=\left(n_{k}, i\right)$ is the first pair which satisfies (17).

All computations have been carried out in double-precision arithmetic within the Turbo Pascal programming framework, on a Pentium-based personal computer. The accuracy and (empirical) stability of the method are witnessed by the fact that the actual errors are always below the prescribed tolerance; the errors have been computed by comparison with the values obtained by the numerical summation tool NSum of Mathematica [12, §3.9.4].

Tables I-VIII refer to the summation of

$$
\sum_{j=1}^{\infty} z^{j} j^{\nu-1} \frac{j^{s}+\cdots+1}{j^{t}+\cdots+1}, z=e^{i \omega \pi / 2}, \omega \in[0,2],
$$

for various admissible quadruples $\nu, s, t, \omega$ corresponding to very slow convergence. Observe that the algorithm behavior gets worse in the neighborhood of $z=1$ (i.e., for $\omega$ "small"), while it works satisfactorily at $z=1(\omega=0)$; as already pointed out in [3], this could be ascribed to the discontinuity of the coefficients $b_{k}(z)$ at $z=1$, since $b_{k}(z) \sim$ const $/(z-1)^{k+2}$ as $z \rightarrow 1$, cf. (4)-(6) and [3] Remark 1.4].

In Tables IX-X we report the results corresponding to the summation of

$$
\sum_{j=1}^{\infty} j^{\nu-1} \frac{\alpha_{1} j+\alpha_{0}}{\beta_{2} j^{2}+\beta_{0}}
$$

for $\nu=1 / 2$ and $\nu=9 / 10$, varying the relative weights of the coefficients of the rational function within a range of two orders of magnitude. Only the values of these coefficients which are not related by a scaling of the series have been considered. 
TABle I. Summation of (19) for $\nu=1, s=1, t=2$

\begin{tabular}{||c||c|c|c|c|c|c|c|c|c||}
\hline$\omega$ & 2 & 1 & 0.8 & 0.6 & 0.4 & 0.2 & 0.1 & 0.05 & 0.01 \\
\hline \hline$n$ & 67 & 90 & 90 & 117 & 149 & 230 & 336 & 547 & 2246 \\
$m$ & 9 & 9 & 10 & 10 & 10 & 11 & 14 & 17 & 21 \\
\hline
\end{tabular}

TABLE II. Summation of (19) for $\nu=1, s=1, t=3$

\begin{tabular}{||c||c|c|c|c|c|c|c|c|c|c||}
\hline$\omega$ & 2 & 1 & 0.8 & 0.6 & 0.4 & 0.2 & 0.1 & 0.05 & 0.01 & 0 \\
\hline \hline$n$ & 60 & 81 & 81 & 105 & 134 & 167 & 300 & 490 & 2010 & 54 \\
$m$ & 8 & 9 & 9 & 9 & 10 & 13 & 13 & 16 & 17 & 8 \\
\hline
\end{tabular}

TABLE III. Summation of (19) for $\nu=1, s=9, t=10$

\begin{tabular}{||c||c|c|c|c|c|c|c|c|c||}
\hline$\omega$ & 2 & 1 & 0.8 & 0.6 & 0.4 & 0.2 & 0.1 & 0.05 & 0.01 \\
\hline \hline$n$ & 31 & 52 & 65 & 65 & 97 & 138 & 253 & 468 & 2027 \\
$m$ & 10 & 11 & 11 & 13 & 13 & 17 & 18 & 20 & 27 \\
\hline
\end{tabular}

TABLE IV. Summation of (19) for $\nu=1, s=9, t=11$

\begin{tabular}{||c||c|c|c|c|c|c|c|c|c|c||}
\hline$\omega$ & 2 & 1 & 0.8 & 0.6 & 0.4 & 0.2 & 0.1 & 0.05 & 0.01 & 0 \\
\hline \hline$n$ & 29 & 48 & 48 & 60 & 73 & 127 & 203 & 386 & 1516 & 16 \\
$m$ & 10 & 10 & 12 & 11 & 13 & 13 & 17 & 15 & 16 & 9 \\
\hline
\end{tabular}

TABLE V. Summation of (19) for $\nu=1 / 2, s=1, t=2$

\begin{tabular}{||c||c|c|c|c|c|c|c|c|c|c||}
\hline$\omega$ & 2 & 1 & 0.8 & 0.6 & 0.4 & 0.2 & 0.1 & 0.05 & 0.01 & 0 \\
\hline \hline$n$ & 40 & 52 & 65 & 65 & 97 & 138 & 253 & 468 & 2027 & 20 \\
$m$ & 10 & 11 & 11 & 13 & 12 & 16 & 17 & 18 & 20 & 11 \\
\hline
\end{tabular}

TABle VI. Summation of (19) for $\nu=1 / 2, s=1, t=3$

\begin{tabular}{||c||c|c|c|c|c|c|c|c|c|c||}
\hline$\omega$ & 2 & 1 & 0.8 & 0.6 & 0.4 & 0.2 & 0.1 & 0.05 & 0.01 & 0 \\
\hline \hline$n$ & 30 & 49 & 49 & 62 & 76 & 133 & 211 & 401 & 1693 & 19 \\
$m$ & 10 & 10 & 12 & 12 & 14 & 14 & 20 & 18 & 19 & 11 \\
\hline
\end{tabular}

As a confirmation of the robustness of the summation algorithm, it is also interesting to quote the following result. For $\alpha_{1}=1, \alpha_{0}=1, \beta_{2}=1$, and $\beta_{0}=10^{4}$ we obtained $(n, m)=(198,37)$ with reltol $=10^{-14}$, and $(n, m)=(119,23)$ with reltol $=10^{-4}$, both starting from $n_{0}=0, S_{n_{0}}=0$ : observe that $n>n^{*}=\tilde{n}=100$ for two very different values of the relative tolerance. 
TABle VII. Summation of (19) for $\nu=1 / 2, s=9, t=10$

\begin{tabular}{||c||c|c|c|c|c|c|c|c|c|c||}
\hline$\omega$ & 2 & 1 & 0.8 & 0.6 & 0.4 & 0.2 & 0.1 & 0.05 & 0.01 & 0 \\
\hline \hline$n$ & 31 & 39 & 48 & 58 & 83 & 133 & 224 & 428 & 1909 & 13 \\
$m$ & 10 & 13 & 13 & 14 & 14 & 17 & 21 & 21 & 25 & 12 \\
\hline
\end{tabular}

TABle VIII. Summation of (19) for $\nu=1 / 2, s=9, t=11$

\begin{tabular}{||c||c|c|c|c|c|c|c|c|c|c||}
\hline$\omega$ & 2 & 1 & 0.8 & 0.6 & 0.4 & 0.2 & 0.1 & 0.05 & 0.01 & 0 \\
\hline \hline$n$ & 30 & 38 & 47 & 57 & 68 & 112 & 119 & 379 & 1653 & 12 \\
$m$ & 10 & 12 & 12 & 12 & 15 & 19 & 17 & 21 & 19 & 9 \\
\hline
\end{tabular}

TABLE IX. Summation of (20) for $\nu=1 / 2$

\begin{tabular}{||c|c|c|c|c|c|c|c|c|c|c|c|c|c||}
\hline$\alpha_{1}$ & 1 & 1 & 1 & 1 & 1 & 1 & 1 & 1 & 1 & 1 & 10 & 10 & 10 \\
$\alpha_{0}$ & 1 & 1 & 1 & 1 & 1 & 10 & 10 & 10 & 10 & 10 & 1 & 1 & 1 \\
$\beta_{2}$ & 1 & 1 & 1 & 10 & $10^{2}$ & 1 & 1 & 1 & 10 & $10^{2}$ & 1 & 1 & 1 \\
$\beta_{0}$ & 1 & 10 & $10^{2}$ & 1 & 1 & 1 & 10 & $10^{2}$ & 1 & 1 & 1 & 10 & $10^{2}$ \\
\hline$n$ & 21 & 32 & 50 & 18 & 10 & 21 & 28 & 50 & 18 & 18 & 21 & 32 & 50 \\
$m$ & 11 & 13 & 18 & 10 & 10 & 11 & 13 & 18 & 10 & 10 & 11 & 12 & 18 \\
\hline
\end{tabular}

\begin{tabular}{||c|c|c|c|c|c|c|c|c|c|c|c|c||}
\hline$\alpha_{1}$ & 10 & 10 & 1 & 1 & 1 & 1 & 1 & $10^{2}$ & $10^{2}$ & $10^{2}$ & $10^{2}$ & $10^{2}$ \\
$\alpha_{0}$ & 1 & 1 & $10^{2}$ & $10^{2}$ & $10^{2}$ & $10^{2}$ & $10^{2}$ & 1 & 1 & 1 & 1 & 1 \\
$\beta_{2}$ & 10 & $10^{2}$ & 1 & 1 & 1 & 10 & $10^{2}$ & 1 & 1 & 1 & 10 & $10^{2}$ \\
$\beta_{0}$ & 1 & 1 & 1 & 10 & $10^{2}$ & 1 & 1 & 1 & 10 & $10^{2}$ & 1 & 1 \\
\hline$n$ & 18 & 18 & 21 & 32 & 50 & 18 & 18 & 21 & 32 & 50 & 18 & 18 \\
$m$ & 9 & 9 & 11 & 13 & 18 & 10 & 10 & 11 & 12 & 18 & 9 & 9 \\
\hline
\end{tabular}

Table X. Summation of (20) for $\nu=9 / 10$

\begin{tabular}{||c|c|c|c|c|c|c|c|c|c|c|c|c|c||}
\hline$\alpha_{1}$ & 1 & 1 & 1 & 1 & 1 & 1 & 1 & 1 & 1 & 1 & 10 & 10 & 10 \\
$\alpha_{0}$ & 1 & 1 & 1 & 1 & 1 & 10 & 10 & 10 & 10 & 10 & 1 & 1 & 1 \\
$\beta_{2}$ & 1 & 1 & 1 & 10 & $10^{2}$ & 1 & 1 & 1 & 10 & $10^{2}$ & 1 & 1 & 1 \\
$\beta_{0}$ & 1 & 10 & $10^{2}$ & 1 & 1 & 1 & 10 & $10^{2}$ & 1 & 1 & 1 & 10 & $10^{2}$ \\
\hline$n$ & 21 & 32 & 50 & 18 & 18 & 21 & 28 & 50 & 18 & 18 & 21 & 32 & 50 \\
$m$ & 10 & 13 & 18 & 10 & 10 & 11 & 13 & 18 & 10 & 10 & 11 & 12 & 18 \\
\hline
\end{tabular}

\begin{tabular}{||c|c|c|c|c|c|c|c|c|c|c|c|c||}
\hline$\alpha_{1}$ & 10 & 10 & 1 & 1 & 1 & 1 & 1 & $10^{2}$ & $10^{2}$ & $10^{2}$ & $10^{2}$ & $10^{2}$ \\
$\alpha_{0}$ & 1 & 1 & $10^{2}$ & $10^{2}$ & $10^{2}$ & $10^{2}$ & $10^{2}$ & 1 & 1 & 1 & 1 & 1 \\
$\beta_{2}$ & 10 & $10^{2}$ & 1 & 1 & 1 & 10 & $10^{2}$ & 1 & 1 & 1 & 10 & $10^{2}$ \\
$\beta_{0}$ & 1 & 1 & 1 & 10 & $10^{2}$ & 1 & 1 & 1 & 10 & $10^{2}$ & 1 & 1 \\
\hline$n$ & 18 & 18 & 24 & 32 & 55 & 18 & 18 & 21 & 28 & 50 & 18 & 15 \\
$m$ & 9 & 9 & 11 & 13 & 18 & 10 & 10 & 11 & 12 & 18 & 9 & 9 \\
\hline
\end{tabular}




\section{ACKNOWLEDGEMENT}

The authors wish to thank Professor Walter Gautschi for a careful reading of the manuscript and valuable suggestions.

\section{REFERENCES}

[1] P. Baratella and B. Gabutti, Uniform approximation on unit disk of series arising in plate contact problems, J. Comput. Appl. Math. 62 (1995), 181-196. MR 96h:65003

[2] S. Dassiè, Convergence acceleration of power series by means of asymptotic summation and extrapolation (Italian), Laurea Thesis, University of Padova, 1995 (advisors: M. Vianello and R. Zanovello).

[3] S. Dassiè, Marco Vianello and Renato Zanovello, Asymptotic summation of power series, Numer. Math. 80 (1998), 61-73. CMP 98:17

[4] W. Gautschi, A class of slowly convergent series and their summation by Gaussian quadrature, Math. Comp. 57 (1991), 309-324. MR 91j:65049

[5] W. Gautschi, On certain slowly convergent series arising in plate contact problems, Math. Comp. 57 (1991), 325-338. MR 91j:40002

[6] W. Gautschi, Orthogonal polynomials: applications and computation, Acta Numerica 1996, Cambridge University Press, Cambridge, 1996, pp. 45-119. CMP 98:14

[7] P. Henrici, Applied and computational complex analysis, Vol. 1, Wiley, New York, 1984. MR 51:8378

[8] S. Lewanowicz, A simple approach to the summation of certain slowly convergent series, Math. Comp. 63 (1994), 741-745. MR 95a:65010

[9] G.V. Milovanović, Summation of series and Gaussian quadratures, Approximation and Computation (R.V.M. Zahar, ed.), ISNM vol.119, Birkhäuser, Basel, 1994, pp. 459-475. MR 96c:65041

[10] G.V. Milovanović, Summation of series and Gaussian quadratures. II, Numer. Algorithms 10 (1995), 127-136. MR 96f:65031

[11] A. Sidi, Analysis of convergence of the T-transformation for power series, Math. Comp. 35 (1980), 833-850. MR 83d:41039

[12] S. Wolfram, Mathematica: A system for doing mathematics by computer, 2nd Edition, Addison-Wesley, Reading, 1991.

Dipartimento di Matematica Pura e Applicata, Università di Padova, Via Belzoni 7, 35131 PAdOVA, ITALY

E-mail address: marcov@math.unipd.it

E-mail address: zanov@math.unipd.it 